\title{
A split-face study of moisturizer containing Centella asiatica extract after ablative fractional carbon dioxide laser resurfacing
}

\author{
Moon Seok Kang ${ }^{1}$, \\ Kyong Chan Park ${ }^{2}$, \\ Seung Min Nam ${ }^{2}$ \\ ${ }^{1}$ WONJIN Plastic Surgery Clinics, Seoul; \\ ${ }^{2}$ Department of Plastic and Reconstructive \\ Surgery, Soonchunhyang University \\ College of Medicine, Bucheon, Korea
}

This work was supported by the Soonchunhyang University Research Fund.
Background Ablative fractional $\mathrm{CO}_{2}$ laser treatment is popular for improving atrophic acne scars; however, complications after laser treatment are unavoidable. The purpose of this study was to investigate the efficacy and safety of a moisturizer containing Centella asiatica extract when applied after ablative fractional $\mathrm{CO}_{2}$ laser resurfacing. Methods A split-face study was conducted between September 2020 and December 2020 on 34 patients ( 16 females and 18 males) who were treated with ablative fractional $\mathrm{CO}_{2}$ laser resurfacing. After laser treatment, $\mathrm{C}$. asiatica extract was applied to the right side of the face and purified water was applied to the left side. We compared the right and left sides of the face at various time points after laser treatment using an automatic skin analysis device to investigate the degree of moisture and post-inflammatory hyperpigmentation.

Results No significant difference in the degree of moisture was found between the two sides of the face immediately after laser treatment $(\mathrm{P}=0.059)$, but there was a significant difference at 1 and 4 hours after post-laser treatment care $(P<0.001)$. Regarding post-inflammatory hyperpigmentation, there was no significant difference between the two sides of the face before laser treatment $(P=0.184)$, but a significant difference was found at 6 weeks after laser treatment $(P<0.001)$.

Conclusions Our results show that applying $C$. asiatica extract is a novel post-laser treatment modality that can provide satisfactory results without complications after ablative fractional $\mathrm{CO}_{2}$ laser resurfacing.

Keywords Lasers / Hyperpigmentation / Moisturizer

\section{INTRODUCTION}

Ablative fractional 10,600 $\mathrm{nm} \mathrm{CO}$ laser resurfacing is a popular method of treating photoaging, skin laxity, and atrophic scars [1-5].

Received: Mar 12, 2021 Revised: Mar 18, 2021 Accepted: Mar 19, 2021 Correspondence: Seung Min Nam Department of Plastic and Reconstructive Surgery, Soonchunhyang University College of Medicine, 170 Jomaru-ro, Wonmi-gu, Bucheon 14584, Korea

Tel: +82-32-621-5311, Fax: +82-32-621-5316, E-mail: zodiac1003@schmc. ac.kr

Copyright @ 2021 The Korean Society for Aesthetic Plastic Surgery.

This is an Open Access article distributed under the terms of the Creative Commons Attribution Non-Commercial License (https://creativecommons.org/licenses/by-nc/4.0/) which permits unrestricted non-commercial use, distribution, and reproduction in any medium, provided the original work is properly cited. $\quad$ www.e-aaps.org
The mechanism of the ablative fractional $\mathrm{CO}_{2}$ laser is fractional photothermolysis with an ablative $10,600 \mathrm{~nm}$ wavelength. It delivers microthermal zones, which are microscopic columns of thermal injury, to a specific fraction of epidermal and dermal tissue $[6,7]$. The microthermal zones are surrounded by healthy, intact skin, and rapid reepithelization occurs via the migration of cells from the adjacent epidermis and follicular units $[1,6,7]$. This rapid reepithelization leads to decreased recovery time, side effects, and complications compared to traditional non-fractional laser resurfacing, but recovery time and downtime following ablative fractional $\mathrm{CO}_{2}$ laser resurfacing are often unavoidable $[8,9]$. Moreover, post-inflammatory hyperpigmentation (PIH) commonly occurs in Asian patients $[8,10]$.

Numerous methods have been introduced to shorten the downtime and the incidence of PIH, such as early sun protection, topical 
or systemic steroids, and moisturizers with anti-inflammatory agents $[3,11]$. However, some studies have reported that the application of post-treatment topical steroids could have unfavorable effects on wound healing and increase the incidence of skin infection and acneiform eruption $[10,12,13]$. Centella asiatica has been used in folk medicine for hundreds of years, and it is traditionally used to improve the condition of small wounds $[14,15]$. It is also a well-known anti-inflammatory and moisturizing agent $[14,15]$.

The aim of our study, therefore, was to evaluate the efficacy and safety of a moisturizer containing $C$. asiatica extract when applied after ablative fractional $\mathrm{CO}_{2}$ laser resurfacing.

\section{METHODS}

A split-face study was conducted to evaluate the efficacy and safety of a moisturizer containing $C$. asiatica extract after ablative fractional $\mathrm{CO}_{2}$ laser resurfacing. Of the patients who underwent ablative fractional $\mathrm{CO}_{2}$ laser resurfacing to treat atrophic acne scars between September 2020 and December 2020, 34 (Fitzpatrick skin type IIIIV) who understood and agreed to the purpose and methods of this study were enrolled. Exclusion criteria included keloid scarring, recent use of oral retinoids, pregnancy, immunosuppressive drug use, active systemic or local infections, local skin disease that might alter wound healing, and a history of psychiatric illness.

The study conformed to the principles of the Declaration of Helsinki, and written consent was obtained from each patient for both the surgery and the publication of photographs of the results. The study was approved by the Institutional Review Board of Soonchunhyang University Bucheon Hospital (IRB No. 2021-02-016).

\section{Laser treatment}

Before laser treatment, topical anesthetic cream containing 2.5\% $(\mathrm{w} / \mathrm{v})$ lidocaine hydrochloric acid and $2.5 \%(\mathrm{w} / \mathrm{v})$ prilocaine (a eutectic mixture of local anesthetics cream; AstraZeneca AB, Södertälje, Sweden) was applied under occlusion for 45 minutes prior to laser treatment. All patients were treated using a 10,600-nm ablative $\mathrm{CO}_{2}$ fractional laser (UltraPulse; Lumenis Ltd., Yokneam, Israel) with a size of $10 \times 10 \mathrm{~mm}$ (with a $5 \%$ overlap) delivered at a fluence of $10.0 \mathrm{~mJ} / \mathrm{cm}^{2}$ in $1.00-\mathrm{ms}$ pulses at $300 \mathrm{~Hz}$ in the Deep FX mode. All laser safety precautions were followed. After treatment, patients were instructed to clean their faces with mild soap and water daily and to apply full-spectrum sunscreen liberally until the end of the study.

\section{Post-laser treatment care}

Each side of the face was treated using different post-treatment methods. A moisturizer containing C. asiatica extract (Kineff 3 Derma Cica Ampoule; Holians Corp., Seoul, Korea) was applied to the right side of the face, and purified water was applied to the left side. Individual sterile cotton buds were used to apply the agents to each side of the face to prevent contamination. After the two agents were applied, a moisture mask (Kineff Hydracica Calming Mask; Holians Corp.) was applied for 30 minutes. The patients were instructed to apply C. asiatica extract (Kineff 3 Derma Cica Ampoule) to the right side of the face and purified water to the left side until removal of the skin crust.

\section{Outcome evaluation}

We evaluated the degree of moisture on the face using an automatic skin analysis device (Mark-Vu; PSI Plus Co., Suwon, Korea) immediately after laser treatment (T0), at post-laser treatment care (T1), 1 hour after exposure to room temperature and moisture after post-laser treatment care (T2), and 4 hours after post-laser treatment care (T3). To evaluate PIH, we used an automatic skin analysis system with ultraviolet light at pretreatment and 6 weeks after treatment.

\section{Statistical analysis}

Statistical analyses were performed using SPSS version 20.0 (IBM Corp., Armonk, NY, USA). The Wilcoxon signed-rank test was used to compare the degree of moisture at T0, T1, T2, and T3 and the degree of PIH at pretreatment and 6 weeks after treatment. Statistical significance was set at $\mathrm{P}<0.05$.

\section{RESULTS}

Of the 34 patients with atrophic acne scars who were treated using ablative fractional $\mathrm{CO}_{2}$ laser resurfacing, 16 were female and 18 were male. The mean age of the patients was 32.4 years (range, 2443 years), and the mean follow-up period was 7 weeks (range, 6-8 weeks) (Table 1). The most frequent complications were erythema, edema, and pain, but infection was not observed during the followup period.

Using a split-face study, we analyzed the differences in the degree of moisture at various time points after laser treatment. The degree of moisture on the right side of the face was 33 (interquartile range [IQR], 32-35) and that on the left side was 33 (IQR, 3234 ) at T0; this difference was not significant. However, the degree of moisture on the right and left sides was 57 (IQR, 56-58) and 52 (IQR, 51-53) at T1, 47 (IQR, 46-48) and 41 (IQR, 40-41) at T2, and 41 (IQR, 40-42) and 34 (IQR, 33-35) at T3, respectively; these differences were significant $(\mathrm{P}<0.001)$ (Fig. 1).

Table 1. Demographic characteristics of patients

\begin{tabular}{lc}
\hline Variable & Value \\
\hline No. of patients & 34 \\
Male sex & 18 \\
Mean age (range, yr) & $32.4(24-43)$ \\
\hline
\end{tabular}




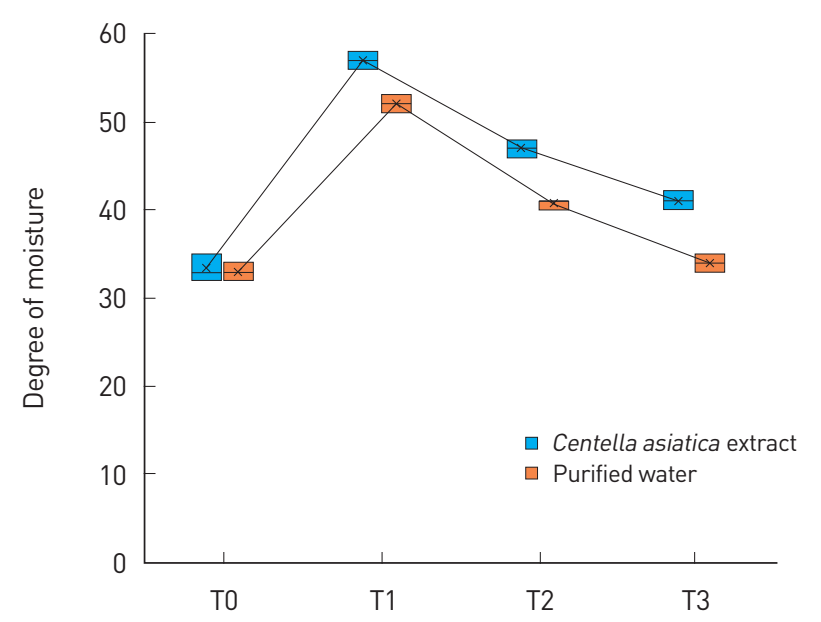

Fig. 1. The degree of moisture was investigated at various time points after ablative fractional $\mathrm{CO}_{2}$ laser treatment. There was no significant difference between the right side of the face (to which Centella asiatica extract was applied) and the left side of the face (to which purified water was applied) immediately post-laser treatment (TO) $(P=0.059)$. There was a significant difference between the right and left sides at post-laser treatment care (T1) and 1 (T2) and 4 hours (T3) after post-laser treatment care $(P<0.001)$.

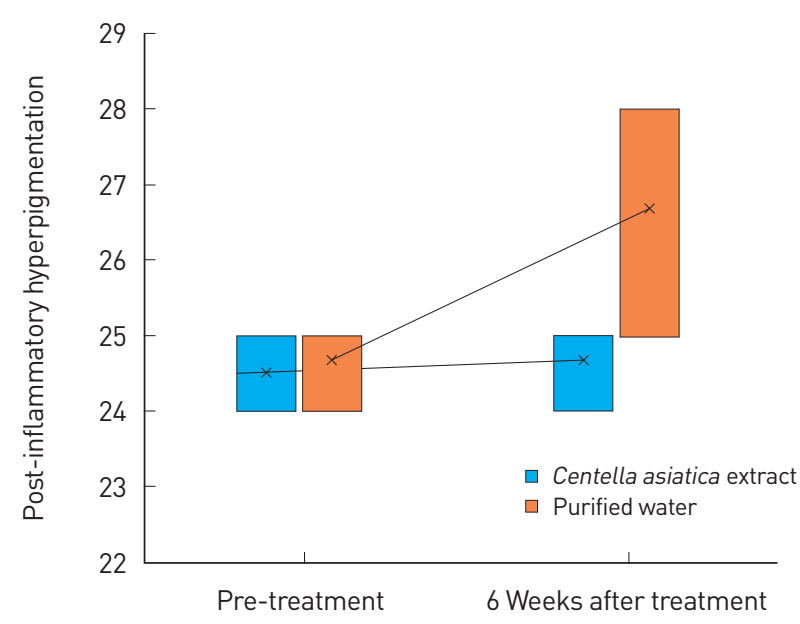

Fig. 2. Post-inflammatory hyperpigmentation was investigated at various time points after ablative fractional $\mathrm{CO}_{2}$ laser treatment. There was no significant difference between the right side of the face (to which Centella asiatica extract was applied) and the left side of the face (to which purified water was applied) at pretreatment $(P=0.184)$. A significant difference was found between the right and left sides 6 weeks after treatment $(P<0.001)$.

In addition, we evaluated the difference in $\mathrm{PIH}$ between the right and left sides of the face. Pretreatment, the degree of PIH was 24.5 on the right side of the face (IQR, 24-25) and 25 on the left side (IQR, 24-25), and the difference was not significant. However, the
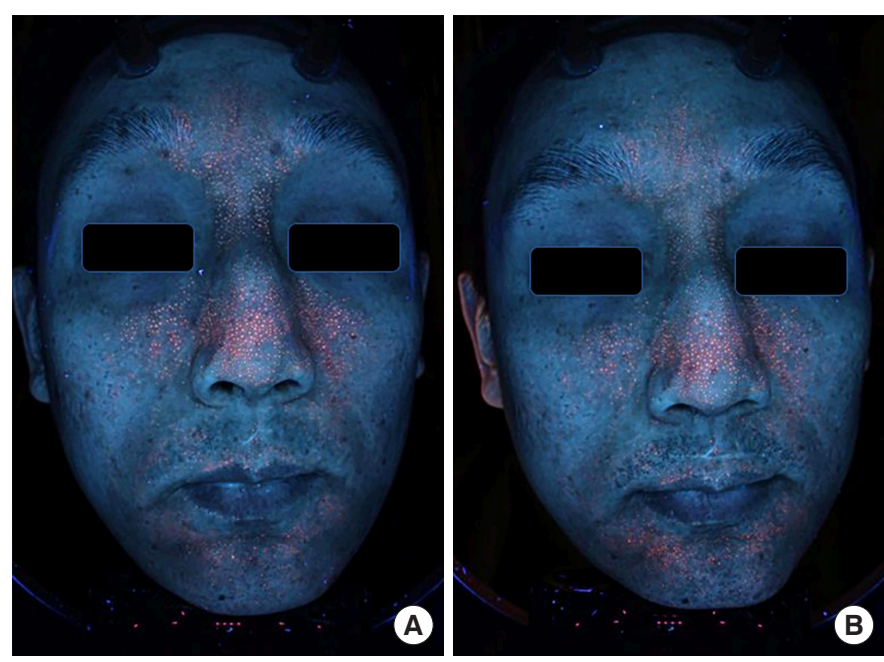

Fig. 3. A 28-year-old male patient with atrophic acne scar. We analyzed post-inflammatory hyperpigmentation (PIH) using an automatic skin analysis device (Mark-Vu). At pretreatment, the degree of PIH was 24 on the right side of the face (to which Centella asiatica extract was applied) and 24.5 on the left side of the face (to which purified water was applied) (A). The degree of PIH was 25 for the right side of the face and 26.5 for the left side (B).
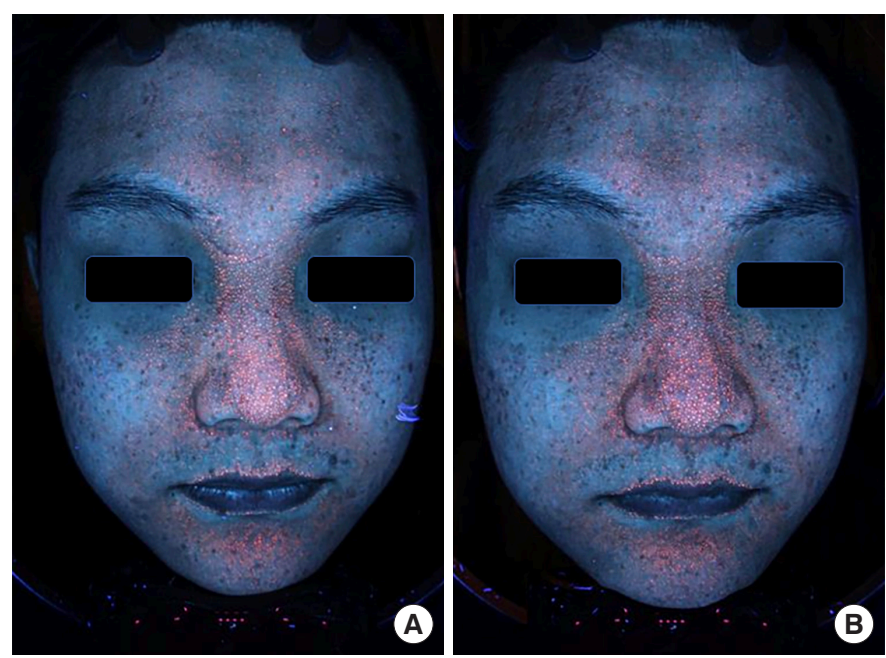

Fig. 4. A 26-year-old male patient with atrophic acne scar. At pretreatment, the degree of post-inflammatory hyperpigmentation (PIH) was 25 on the right side of the face (to which Centella asiatica extract was applied) and 25 on the left side of the face (to which purified water was applied) (A). The degree of PIH was 25 for the right side of the face and 27 for the left side (B).

degree of PIH was 25 on the right side of the face (IQR, 24-25) and 27 on the left side (IQR, 25-28) at 6 weeks after laser treatment, which was a significant difference $(\mathrm{P}<0.001)$ (Figs. 2-4). 


\section{DISCUSSION}

Although ablative fractional $\mathrm{CO}_{2}$ laser resurfacing can achieve rapid wound healing compared to traditional ablative lasers, it often causes complications associated with wound healing. Wound healing is a complex and dynamic process that can be divided into three phases: inflammation, proliferation, and remodeling $[3,16]$. In particular, a moist wound environment can provide sufficient and rapid wound healing and avoid post-laser complications, such as infection, hypertrophic scarring, and PIH $[8,16]$.

C. asiatica is a medical plant that has been used for at least 3000 years $[14,15]$. It contains pentacyclic triterpenes, such as asiaticoside, madecassoside, asiatic acid, and madecassic acid, as its active compounds [14]. Tenni et al. [17] reported a significant increase in the percentage of collagen and fibronectin of a human skin fibroblast after the application of C. asiatica extract. In addition, C. asiatica extract increased collagen synthesis in a dose-dependent manner [18]. C. asiatica extract could facilitate scar maturation by increasing type I collagen synthesis and the type I to type III collagen ratio [19], and it could prevent keloid scar formation by negatively regulating the expression of the transforming growth factor (TGF)$\beta I$ and TGF- $\beta$ II receptor genes and positively regulating the expression of $S m a d 7$ [20].

In our study, we evaluated the degree of moisture during postlaser treatment care using a split-face study. The degree of moisture on the right side of the face to which $C$. asiatica extract was applied post-laser treatment care was higher than that on the left side to which purified water was applied. In addition, we investigated the degree of moisture at 1 and 4 hours after post-laser treatment care; the ride side of the face maintained more moisture than the left side until 4 hours after post-laser treatment care. The differences in moisture between the two sides of the face were significant at postlaser treatment care and at 1 and 4 hours after post-laser treatment care. To investigate PIH, we evaluated the degree of $\mathrm{PIH}$ at pretreatment and 6 weeks after treatment using a split-face study. The patients were instructed to apply $C$. asiatica extract to the right side of the face and purified water to the left side until the skin crusts were removed. At 6 weeks after treatment, the degree of $\mathrm{PIH}$ on the right side of the face was significantly lower than that on the left side. Rapid wound healing would provide a moist environment, and rapid wound healing after laser treatment could provide satisfactory results without complications $[8,13,16]$. Our study results show that applying $C$. asiatica extract to the face after ablative fractional $\mathrm{CO}_{2}$ laser treatment can provide a moist wound environment and reduce the degree of PIH. Moreover, the results on the effects of $C$. asiatica extract were obtained using a split-face study to eliminate patient bias.

Therefore, the application of $C$. asiatica extract could provide a novel post-laser treatment modality to decrease the incidence of $\mathrm{PIH}$ and promote rapid wound healing while maintaining a moist wound environment. Nevertheless, further study is recommended because our study has some limitations, including a small sample size, short-term follow-up period, and the inclusion of only an Asian population with Fitzpatrick skin types III-IV.

Although further studies with long-term follow-up periods are needed, in this study, the application of C. asiatica extract provided a moist wound environment to induce rapid wound healing and prevent $\mathrm{PIH}$ after ablative fractional $\mathrm{CO}_{2}$ laser resurfacing.

\section{NOTES}

\section{Conflict of interest}

No potential conflict of interest relevant to this article was reported.

\section{Ethical approval}

The study was approved by the Institutional Review Board of Soonchunhyang University Bucheon Hospital (IRB No. 2021-02-016) and performed in accordance with the principles of the Declaration of Helsinki.

\section{Patient consent}

The patients provided written informed consent for the publication and the use of their images.

\section{ORCID}

Moon Seok Kang

https://orcid.org/0000-0001-7072-6091

Kyong Chan Park https://orcid.org/0000-0002-0742-1233

Seung Min Nam https://orcid.org/0000-0001-7865-5013

\section{REFERENCES}

1. Chang YC, Croix J, Hernandez S, et al. A comparative split-face trial of plant-based hypoallergenic ointment vs petroleum-based ointment following fractionated carbon dioxide laser resurfacing of the face. J Drugs Dermatol 2018;17:1178-82.

2. Ho C, Nguyen Q, Lowe NJ, et al. Laser resurfacing in pigmented skin. Dermatol Surg 1995;21:1035-7.

3. Techapichetvanich $\mathrm{T}$, Wanitphakdeedecha $\mathrm{R}$, Iamphonrat $\mathrm{T}$, et al. The effects of recombinant human epidermal growth factor containing ointment on wound healing and post inflammatory hyperpigmentation prevention after fractional ablative skin resurfacing: a split-face randomized controlled study. J Cosmet Dermatol 2018;17:756-61.

4. Tierney EP, Hanke CW, Watkins L. Treatment of lower eyelid rhytids and laxity with ablative fractionated carbon-dioxide laser resurfacing: case series and review of the literature. J Am Acad Dermatol 2011;64: 730-40.

5. Weiss ET, Chapas A, Brightman L, et al. Successful treatment of atrophic postoperative and traumatic scarring with carbon dioxide ablative fractional resurfacing: quantitative volumetric scar improvement. Arch Dermatol 2010;146:133-40. 
6. Abou Eitta RS, Ismail AA, Abdelmaksoud RA, et al. Evaluation of autologous adipose-derived stem cells vs. fractional carbon dioxide laser in the treatment of post acne scars: a split-face study. Int J Dermatol 2019;58:1212-22.

7. Hunzeker CM, Weiss ET, Geronemus RG. Fractionated CO2 laser resurfacing: our experience with more than 2000 treatments. Aesthet Surg J 2009;29:317-22.

8. Lueangarun S, Srituravanit A, Tempark T. Efficacy and safety of moisturizer containing $5 \%$ panthenol, madecassoside, and copper-zincmanganese versus $0.02 \%$ triamcinolone acetonide cream in decreasing adverse reaction and downtime after ablative fractional carbon dioxide laser resurfacing: a split-face, double-blinded, randomized, controlled trial. J Cosmet Dermatol 2019;18:1751-7.

9. Manuskiatti W, Triwongwaranat D, Varothai S, et al. Efficacy and safety of a carbon-dioxide ablative fractional resurfacing device for treatment of atrophic acne scars in Asians. J Am Acad Dermatol 2010;63: 274-83.

10. Cheyasak N, Manuskiatti W, Maneeprasopchoke P, et al. Topical corticosteroids minimise the risk of postinflammatory hyper-pigmentation after ablative fractional CO2 laser resurfacing in Asians. Acta Derm Venereol 2015;95:201-5.

11. Duplechain JK, Rubin MG, Kim K. Novel post-treatment care after ablative and fractional CO2 laser resurfacing. J Cosmet Laser Ther 2014;16:77-82.

12. Graber EM, Tanzi EL, Alster TS. Side effects and complications of frac- tional laser photothermolysis: experience with 961 treatments. Dermatol Surg 2008;34:301-7.

13. Shamsaldeen O, Peterson JD, Goldman MP. The adverse events of deep fractional $\mathrm{CO}(2)$ : a retrospective study of 490 treatments in 374 patients. Lasers Surg Med 2011;43:453-6.

14. Bylka W, Znajdek-Awizen P, Studzinska-Sroka E, et al. Centella asiatica in dermatology: an overview. Phytother Res 2014;28:1117-24.

15. Ratz-Lyko A, Arct J, Pytkowska K. Moisturizing and antiinflammatory properties of cosmetic formulations containing Centella asiatica extract. Indian J Pharm Sci 2016;78:27-33.

16. Francesko A, Petkova P, Tzanov T. Hydrogel dressings for advanced wound management. Curr Med Chem 2018;25:5782-97.

17. Tenni R, Zanaboni G, De Agostini MP, et al. Effect of the triterpenoid fraction of Centella asiatica on macromolecules of the connective matrix in human skin fibroblast cultures. Ital J Biochem 1988;37:69-77.

18. Maquart FX, Bellon G, Gillery P, et al. Stimulation of collagen synthesis in fibroblast cultures by a triterpene extracted from Centella asiatica. Connect Tissue Res 1990;24:107-20.

19. Bonte F, Dumas M, Chaudagne C, et al. Comparative activity of asiaticoside and madecassoside on type I and III collagen synthesis by cultured human fibroblasts. Ann Pharm Fr 1995;53:38-42.

20. Tang B, Zhu B, Liang Y, et al. Asiaticoside suppresses collagen expression and TGF- $\beta /$ Smad signaling through inducing Smad7 and inhibiting TGF- $\beta$ RI and TGF- $\beta$ RII in keloid fibroblasts. Arch Dermatol Res 2011;303:563-72. 